\title{
To compare advantage of non-fixation versus fixation of mesh in Laparoscopic totally Extra Peritoneal (TEP) repair of inguinal hernias
}

\author{
Ambar Gangopadhyay ${ }^{1}$, Bikash Chandra Ghosh ${ }^{2}$ \\ ${ }_{1,2}^{1}$ Associate Professor, Department of General Surgery, R. G. Kar Medical College Kolkata
}

A B S T R A C T

\begin{abstract}
Background: Mesh fixation during laparoscopic totally extra peritoneal repair is thought to be necessary to prevent recurrence of infections and post-operative complications. However, mesh fixation might increase the postoperative complications and chronic pain. This study was to describe the experience of a single surgeon at R.G. Kar Medical College and Hospital performing this operation. This study evaluates the outcomes of laparoscopic hernioplasty performed with and without mesh fixation at our institution. Aims and Objective: To compare advantage of non-fixation versus fixation of mesh in laparoscopic Totally Extra peritoneal (TEP) repair of inguinal hernias. This study was conducted for analysis of outcome with respect to pain, operative time, intra and postoperative complication, days of hospital stay, recurrence regarding the procedure between fixation and non-fixation of mesh in totally extra peritoneal repair of inguinal hernia. Materials and Methods: The study was conducted in the Department of Surgery, R.G.Kar Medical College and Hospital from January 2011 to April 2012. All patients admitted in General surgical unit presenting with uncomplicated unilateral inguinal hernias were included. A total of 60 patients were included in the study, of which 30 patients underwent TEP repair without fixation of mesh and for remaining 30 patients the mesh was fixed using metallic tacks. Results: Difference in average pain score at $24 \mathrm{hrs,}$ 72 hrs, 1 month and 6 months was significant statistically $(p=0.003, p=0.003, p<0.001$ and $p=0.001$ respectively) when compared in both groups. There was no recurrence in the study period in either of the groups. The duration of operative time and days of hospital stay was higher in fixation group and was statistically significant. Conclusion: Mesh fixation appears to be disadvantageous in TEP repair of inguinal hernias compared to non- fixation of mesh as it is associated with higher operative time, higher postoperative complication and an increased likelihood of developing chronic groin pain. The omission of mesh fixation did not increase the risk of early hernia recurrence.
\end{abstract}

Key words: Inguinal hernia; Hernioplasty; Laparoscopic inguinal hernia repair; Totally extra peritoneal repair; Mesh fixation

\section{INTRODUCTION}

With the advent of laparoscopic surgery, it has revolutionized the treatment of hernia. Laparoscopic hernia repair can be done by two methods - Trans Abdominal Pre Peritoneal (TAPP) and Totally Extra Peritoneal (TEP). ${ }^{1}$ Both these methods have their advantages and disadvantages. In TEP mesh is placed in the extra peritoneal space which is fixed by various fixation devices, so that it does not get displaced leading to recurrence of post-operative complications. ${ }^{2}$ Various types of metallic and non-metallic tacks are used. These devices have their own disadvantages like postoperative chronic pain due to nerve injury or entrapment, or pubalgia caused by stapling of prosthesis to Cooper's ligament, bleeding or hematomas in Retzius space. Despite the fact that recent trials $s^{3}$ have confirmed that laparoscopic inguinal hernia repair is comparable with open surgery in terms of outcome and recovery, yet this needs to be widely accepted worldwide. 
Whether non fixation of mesh is superior to fixation of mesh still remain controversial. The main focus of debate surrounds on cost-effectiveness, score of pain, operating time, intra or post-operative complications, recurrence, days of hospital stay after surgical procedure, and above all the quality of life of the patients after surgery. TEP repair is still gaining recognition because many surgeons have become cautious of the potential complications when entering the peritoneal cavity using the trans-abdominal approach. There are entrenched belief within the surgical community that mesh fixation is a vital step in the repair to reduce the risk of mesh folding or migration that could lead to early hernia recurrence. In this study we describe the experience of a single surgeon at our institution performing unilateral laparoscopic TEP repair with and without mesh fixation.

\section{AIMS AND OBJECTIVE}

The aim of the current study was to analysis the outcome of fixation vs non-fixation of mesh in unilateral inguinal hernia male patients with respect to post-operative pain, time of surgical procedure, intra and post-operative complications, days of hospital stay after surgery and recurrence.

\section{MATERIAL AND METHODS}

The study was carried out in the Department of Surgery, R.G.Kar Medical College and Hospital from January 2011 to April 2012.All patients admitted in General surgical unit presenting with uncomplicated unilateral inguinal hernias were included. A total of 60 patients were included in the study, thirty patients underwent TEP repair without fixation of mesh (appendix 1) and in remaining 30 patients mesh was fixed (appendix 2 and 3) using metallic tacks.All cases were between 18 years to 78 years.

Parameters used for comparison between non-fixation and fixation of Mesh were pain, duration of operation, intra and post-operative complication, duration of hospital stay and long term groin pain and recurrence. The Inclusion criteria were uncomplicated hernia, willing for laparoscopic operation and medical fitness. Exclusion criteria were patients who had acute or obstructed inguino-scrotal hernia, recurrent and bilateral hernia, previous history of midline, paramedian or suprapubic incision, undergone totally extra peritoneal repair to open repair and any cases of recurrent hernia, medically unfit were excluded from the study.

\section{Statistical analysis}

In this study the end point data was analysed by two statistical softwares-Statistical package for the social sciences version 17 and Epi info version 4. The categorical data namely; Intra operative and Post-operative complication was analysed by Chi-square test. The Independent sample ' $\mathrm{t}$ ' test was used to compared ordinal data namely age, operating time, post-operative pain and duration of stay in hospital.

\section{Result}

The current study was to compare the advantage of non-fixation and fixation of mesh in laparoscopic TEP repair of inguinal hernia. Of the thirty cases of fixation group 20 cases were Right sided hernias and the rest were Left sided. Of these, 17 cases were direct and rest were indirect hernias. Among the non-fixation group, of thirty cases, 16 cases were right sided and 14 cases were Left sided hernias. Of these, 21 cases were direct and 9 cases were indirect hernias.

Distribution of study subjects is depicted in table no. 1 . From the table, it is understood that inguinal hernia is more common is old age as we observed that, $43.33 \%$ of the cases among the fixation group and $36.67 \%$ in non-fixation group were above 50 years of age (table 1).Comparison of average age of study subjects between groups are shown in table 2.

Mean age in fixation was 44.87 and non-fixation group was 44.20 , where pair $\mathrm{t}$ test $=0.174$ and $\mathrm{p}=0.863$. This shows difference in mean age between two groups was not statistically significant $(\mathrm{p}=0.863)$. So both groups were comparable.

When analysed for post-operative pain among the two groups, it was observed that the VAS score was statistically significant at 24 hours and beyond up to six months as presented in table 3 .

\begin{tabular}{|c|c|c|c|c|}
\hline AGES & $\begin{array}{l}\text { FIXATION } \\
(\mathrm{n} 1=30)\end{array}$ & $\%$ & $\begin{array}{l}\text { NON FIXATION } \\
(\mathrm{n} 2=30)\end{array}$ & $\%$ \\
\hline$<21$ & 2 & 6.67 & 1 & 3.33 \\
\hline $21-30$ & 4 & 13.33 & 5 & 16.67 \\
\hline $31-40$ & 6 & 20 & 8 & 26.67 \\
\hline $41-50$ & 5 & 16.67 & 5 & 16.67 \\
\hline$>50$ & 13 & 43.33 & 11 & 36.67 \\
\hline Total & 30 & 100 & 30 & 100 \\
\hline
\end{tabular}

\begin{tabular}{|c|c|c|c|c|}
\hline & Mean+SD & $\mathbf{t}$ & Df & $\mathbf{P}$ \\
\hline $\begin{array}{l}\text { Fixation } \\
\text { Non fixation }\end{array}$ & $\begin{array}{l}44.87+15.014 \\
44.20+14.684\end{array}$ & 0.174 & 58 & 0.863 \\
\hline
\end{tabular}


Figure-1 depicts operative time of surgery between both the groups. It was observed that, 1 case of fixation and 7 cases of non-fixation groups required operative time less than 40 mins. Furthermore it was observed that the ideal time duration of surgery in case of non-fixation was 41 to 60 mins and in case of fixation it exceeded beyond 80 mins. The mean operative time in fixation group was $95.83( \pm 18.80)$ minutes whereas in case of non-fixation group it was $56.83( \pm 20.49)$ minutes, which was statistically significant $(\mathrm{p}<0.001)$.

When the intraoperative complications were compared between non-fixation vs. fixation it was observed that in addition to complications like bleeding from inferior epigastric vessels, gonadal vessels and pneumoperitonium, fixation group had additive complication namely bleeding from tacker site. In this study, it was noted that there was $23.33 \%$ complications among fixation group whereas only $13.33 \%$ of complications was observed in non-fixation group as shown in table 4 . Though the complications were higher in fixation group, statistical analysis revealed no significant $(\mathrm{p}=0.504)$.

When analysed for post-operative complications, 10\% higher in fixation group (table 5), though the complications were insignificant $(\mathrm{p}=0.504)$.

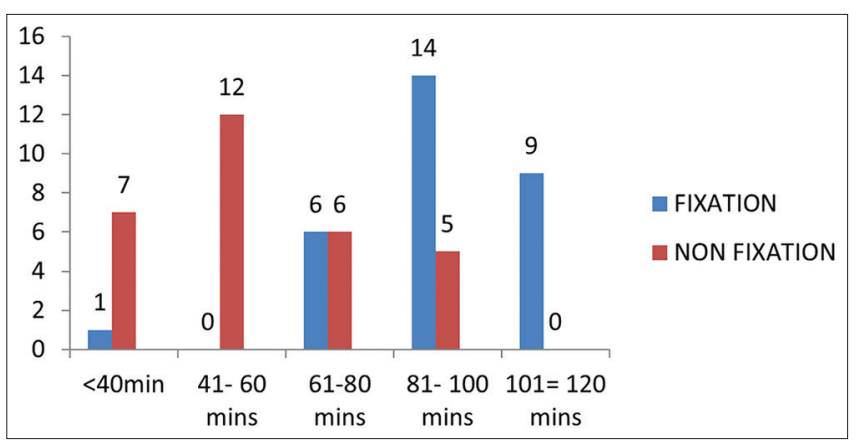

Figure 1: Operative time of surgery
When we analysed the average duration of post-operative hospital stay, the patients in the fixation group had to stay an average of 3.63 days when compared to non-fixation group with an average of 2.93 days, and the difference between the hospital stay was statistically significant $(p<0.001)$ (Table 6).

The study could not observe any recurrence of hernia as duration was slightly higher than one year which is short enough for any recurrence. It is one of the limitations of the study.

\section{DISCUSSION}

Many surgeons who perform TEP appear to hold the unproven belief that mesh fixation is necessary for the prevention of hernia recurrence. At the same time it is widely acknowledged that this need for surgical fixation is only temporary, as tissue incorporation into the mesh, characterized by significant cellular growth by two weeks and collagen deposition within two months, achieves effective permanent fixation. The current study was undertaken to analysis the outcome of fixation vs non-fixation of mesh in unilateral inguinal hernia male patients with respect to post-operative pain, time of surgical procedure, intra and post-operative complications, days of hospital stay after surgery and recurrence.

Our study didn't shows significant difference in pain score at 12 hours of post-operative period, but had significant difference in VAS score at 24 hours, 72 hours, 1 month and 6 months follow up. In a study conducted by Lau $\mathrm{H}$ et al among 313 patients, observed that the chronic pain among ranges from $9 \%$ to $22 \%$ in patients after laparoscopic TEP repair with mesh fixation. ${ }^{4}$ Study conducted among 929 patients to evaluate the incidence of chronic pain after laparoscopic TEP repair with and without mesh fixation observed no significant difference in

\begin{tabular}{|c|c|c|c|c|c|}
\hline \multirow[t]{2}{*}{ Different stage } & \multicolumn{2}{|c|}{ Mean+SD } & \multirow[t]{2}{*}{$\mathbf{t}$} & \multirow[t]{2}{*}{ Df } & \multirow[t]{2}{*}{$\mathbf{P}$} \\
\hline & Fixation & Non fixation & & & \\
\hline $12 \mathrm{hrs}$ & $38.67+18.889$ & $30.00+15.97$ & 1.919 & 58 & 0.060 \\
\hline $24 \mathrm{hrs}$ & $24.33+16.121$ & $12.33+13.817$ & 3.096 & 58 & 0.003 \\
\hline $72 \mathrm{hrs}$ & $13.00+15.347$ & $3.67+6.687$ & 3.054 & 58 & 0.003 \\
\hline 1 month & $7.33+10.807$ & 000 & 3.717 & 58 & $<0.001$ \\
\hline 6 month & $4.33+6.789$ & 000 & 3.496 & 58 & 0.001 \\
\hline
\end{tabular}

\begin{tabular}{|c|c|c|c|c|c|c|}
\hline \multirow[t]{2}{*}{ Group } & \multicolumn{2}{|c|}{ No. $(\%)$} & \multirow[t]{2}{*}{$\mathbf{X} 2$} & \multirow[t]{2}{*}{ Df } & \multirow[t]{2}{*}{$p$} & \multirow[t]{2}{*}{ OR } \\
\hline & Complication & No-complication & & & & \\
\hline $\begin{array}{l}\text { Fixation }(n 1=30) \\
\text { Non fixation }(n=30)\end{array}$ & $\begin{array}{l}7(23.33) \\
4(13.33)\end{array}$ & $\begin{array}{l}23(76.67) \\
26(86.67)\end{array}$ & 0.45 & 1 & 0.504 & $1.98<\mathrm{OR}<9.41$ \\
\hline
\end{tabular}


Table 5: Distribution of post-operative complication in study group

\begin{tabular}{|c|c|c|c|c|c|c|}
\hline & \multicolumn{2}{|c|}{ No. (\%) } & \multirow[t]{2}{*}{$\mathbf{X} 2$} & \multirow[t]{2}{*}{ df } & \multirow[t]{2}{*}{$\mathbf{P}$} & \multirow[t]{2}{*}{ OR } \\
\hline & Complication & No complication & & & & \\
\hline Fixation $(\mathrm{n} 1=30)$ & $6(20)$ & $24(80)$ & 0.45 & 1 & 0.504 & $2.25<O R<12.97$ \\
\hline No fixation $\left(n_{2}=30\right)$ & $3(10)$ & $27(90)$ & & & & \\
\hline
\end{tabular}

\begin{tabular}{|c|c|c|c|c|}
\hline & Mean+SD & $T$ & Df & $\mathbf{P}$ \\
\hline Fixation & $3.63+0.556$ & 3.986 & 58 & $<0.001$ \\
\hline Non fixation & $2.93+0.785$ & & & \\
\hline
\end{tabular}

pain score in both groups up to 1 week but they observed significant difference in pain score at 1 month. ${ }^{5}$ In another interventional prospective study based on comparison of fixation vs non-fixation of mesh in total extra peritoneal hernioplasty conducted in Pakistan on 63 patients, the mean pain score in fixation group was $4.7 \pm 0.683$ where as in non-fixation group was $4.1 \pm 0.860$ and the differences in the pain score was highly significant $(\mathrm{p}<0.001) .{ }^{6} \mathrm{In}$ another study conducted in Turkey with an aim to analysis the outcomes of laparoscopic total extra peritoneal(TEP) inguinal hernia repair without using mesh fixation among 60 patients between 2012 and 2015, Only 4 patients had level 2 pain at the end of the first week, and none complained of pain at first, third, and sixth month of follow-up period. ${ }^{7}$ In yet another prospective controlled, randomized study conducted in Egypt with an aim to compare mesh fixation versus non fixation regarding postoperative pain and recurrence rates among 60 patients with inguinal hernias. They observed a significant reduction in postoperative pain and chronic pain in patients without mesh fixation in comparison with patients with mesh fixation and early return to work. The study found mesh non fixation reduces the incidence of postoperative pain and chronic pain in patients undergoing TAPP repair. ${ }^{8}$ In another randomized clinical trial study of fixation vs non fixation of mesh in Total Extraperitoneal Inguinal Hernioplasty conducted in Spain for a period of January 1999 to December 2001 among 170 patients. The study observed the pain score among non-fixation group was $1.65 \pm 1.3$ and $0.14 \pm 1.7$ in post-operative 24 hours and 1 month respectively whereas among the fixation group it was $1.78 \pm 1.4$ and $0.16 \pm 0.6$ in post-operative 24 hours and 1 month though there was no significant difference among the group.'

In the current study we observed that the ideal time duration of surgery in case of non-fixation was 41 to $60 \mathrm{mins}$ and in case of fixation it exceeded beyond $80 \mathrm{mins}$ which was statistically significant. In another randomized clinical trial study among 170 patients, the study observed mean operative time of unilateral non-fixation was $39.1 \pm 15.3$ minutes whereas in unilateral fixation was
$45.7 \pm 17.9$ minutes and the difference between time was significant $(\mathrm{p}=0.01)$ which was similar to the current study.

Though the complications were higher in fixation group, it was statistically insignificant. Similarly in case of post-operative complications, $10 \%$ of the patients with fixation group had more complications but again the results were insignificant. In another interventional prospective study, urinary retention was one of the post operative complications in five patients $(15.6 \%)$ in fixation compared to four patients $(12.9 \%)$ with the similar complication in non-fixations group. ${ }^{6}$ In another study, only 4 patients developed seroma in the first week of post-surgical complication. ${ }^{7}$ In yet another prospective controlled, two patients $(6.7 \%)$ developed seroma among patients undergone fixation though it was non-significant. ${ }^{8}$

In another randomized prospective cohort study, of 274 patients only 19 inguinal seromas (6.1\%) were identified at 2 weeks, only $7(1.9 \%)$ remained at 1 month, and none at 1 year among fixation group though the findings were insignificant. ${ }^{10}$

In our study the average duration of post-operative hospital stay among the patients in the fixation group was 3.63 days when compared to non-fixation group which was 2.93 days, and the study observed statistically significant difference between the two groups. In another study conducted in Turkey with an aim to analysis the outcomes of laparoscopic total extra peritoneal (TEP) inguinal hernia repair without using mesh fixation among 60 patients between 2012 and 2015, all patients were discharged within post-operative 24 hours. ${ }^{7}$

In a study conducted on Bilateral Laparoscopic Totally Extraperitoneal Repair Without Mesh Fixation among 343 patients, the duration of hospital stay after surgery was few hours for $99 \%$ of the patients where as $1 \%$ was discharged by 24 hours. ${ }^{11}$ In a Randomized Prospective Study of Totally Extraperitoneal Inguinal Hernia Repair: Fixation Versus No Fixation of Mesh at Minnesota, USA among 274 patients, the study observed the mean duration of stay in hospital among non-fixation group was 8.3 hours and 16 hours in case of fixation group and the difference among the duration was significant $(\mathrm{p}=0.01) .{ }^{12}$

Our study could not observe any recurrence of hernia as duration was slightly higher than one year which is short enough for any recurrence. In yet another prospective controlled, randomized study conducted in Egypt with an 
aim to compare mesh fixation versus non fixation regarding postoperative pain and recurrence rates among 60 patients with inguinal hernias. The study observed $0 \%$ recurrence among fixation group where as among non fixation group the recurrence rate was 3.3\% within one year follow up period though the differences among the group was not significant. ${ }^{8}$ Yet another study, no recurrence of hernia was observed among both the groups. ${ }^{6,12}$

It was not the intention of the present study to investigate the long-term recurrence rate of TEP. Rather, it was to test the hypothesis that, without fixation, the mesh might move or fold before tissue ingrowth has had an opportunity to occur, and lead to recurrence by the uncovering of hernial defects.

\section{CONCLUSION}

Mesh fixation appears to be unnecessary in TEP repair of inguinal hernias. It is associated with higher operative time, higher postoperative complication and an increased likelihood of developing chronic groin pain and offers no clinical advantages and increases the cost of the process. Our results recommend limiting the use of mesh fixation in the laparoscopic approach to cases of unilateral hernias.

So from the current study it is very much reasonable to conclude that postoperative chronic pain is less likely to develop in patients who undergo TEP repair without mesh fixation; moreover, even when chronic pain does develop in such patients, their pain will be mild and unlikely to necessitate re-exploration.

\section{LIMITATIONS OF THE STUDY}

The limitations of the study includes small study population, single centric study with no female patients, all surgical procedures done by a single surgeon, the post- operative follow up for recurrence is less time duration and above all patients with recurrent and bilateral hernia were not included in this study.

\section{REFERENCES}

1. Bhandarkar DS, Shankar M and Udwadia TE. Laparoscopic surgery for inguinal hernia: Current status and controversies. J Minim Access Surg 2006; 2(3): 178-186.

2. Bittner R, Arregui ME, BisgaardT, Dudai M, Ferzli GS, Fitzgibbons RJ, et al. Guidelines for laparoscopic (TAPP) and endoscopic (TEP) treatment of inguinal Hernia [International Endohernia Society (IEHS)]. Surg Endosc 2011; 25(9): 2773-2843.

3. Heikkinen T, Bringman S, Ohtonen P, Kunelius P, Haukipuro K and Hulkko A. Five-year outcome of laparoscopic and Lichtenstein hernioplasties. Surg Endosc 2004; 18:518-522.

4. Lau H, Patil NG, Yuen WK and Lee F. Prevalence and severity of chronic groin pain after endoscopic totally extraperitoneal inguinal hernioplasty. Surg Endosc 2003; 17:1620 -1623.

5. Taylor C, Layani L, Liew V, Ghusn M, Crampton N and White S. Laparoscopic inguinal hernia repair without mesh fixation, early results of a large randomized clinical trial. Surg Endosc 2008; 22: 757-762.

6. Ayyaz M, Farooka MW, Toor AA, Malik AA, Khokhar HA, Khan A, et al. Mesh fixation vs. non-fixation in total extra peritoneal mesh hernioplasty. J Pak Med Assoc 2015; 65(3):270-272.

7. Sağıroğlu J, Özdemir T, Atak T, Gök MA, Erdoğan KO, Eren T, et al. Laparoscopic Total Extraperitoneal Inguinal Hernia Repair Without Mesh Fixation: Report of Early Outcomes. South Clinlst Euras 2016; 27(3):215-219.

8. Darwish AA and Hegab AA. Tack fixation versus nonfixation of mesh in laparoscopic transabdominal preperitoneal hernia repair. The Egyptian Journal of Surgery 2016; 35:327-331.

9. Moreno-Egea A, Torralba Martínez JA, Cuenca GM and Aguayo Albasini JL. Randomized Clinical Trial of Fixation vs Non fixation of Mesh in Total Extraperitoneal Inguinal Hernioplasty. Arch Surg 2004; 139: 1376-1379.

10. Messaris E, Nicastri $G$ and Dudrick SJ. Total Extraperitoneal Laparoscopic Inguinal Hernia Repair Without Mesh Fixation. Prospective Study With 1-Year Follow-up Results. Arch Surg 2010; 145(4):334-338.

11. Dehal A, Woodward B, Johna S and Yamanishi F. Bilateral Laparoscopic Totally Extraperitoneal Repair Without Mesh Fixation. Journal of the Society of Laparoendoscopic Surgeons 2014; 18(3): e2014.00297.

12. Koch CA, Greenlee SM, Larson DR, Harrington JR and Farley DR. Randomized Prospective Study of Totally Extraperitoneal Inguinal Hernia Repair: Fixation Versus No Fixation of Mesh. Journal of the Society of Laparoendoscopic Surgeons 2006; 10:457-460. 


\section{APPENDIX}

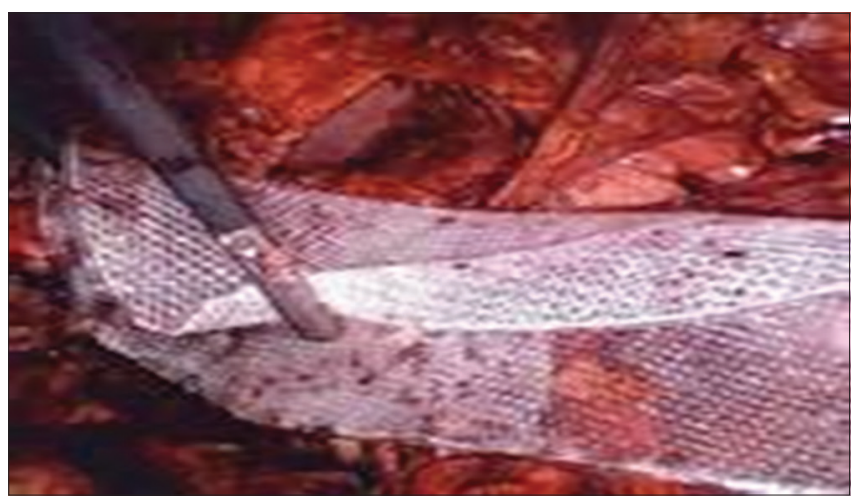

Figure 1: Mesh without fixation

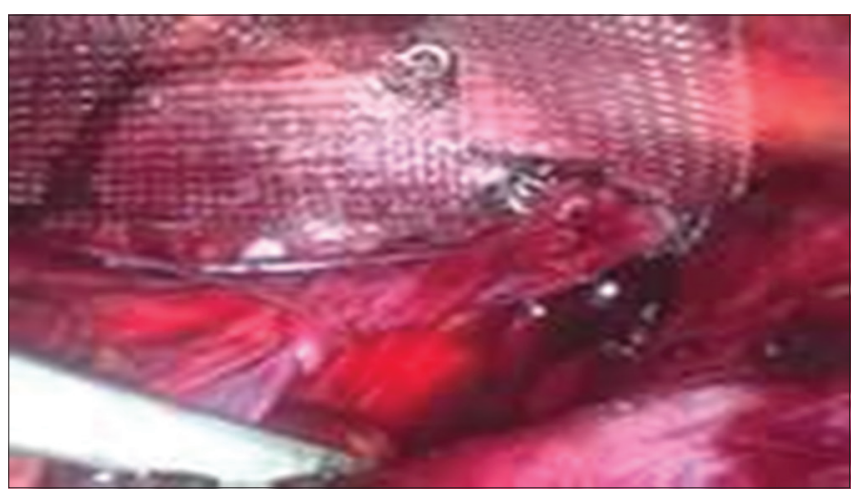

Figure 2: Mesh with fixation

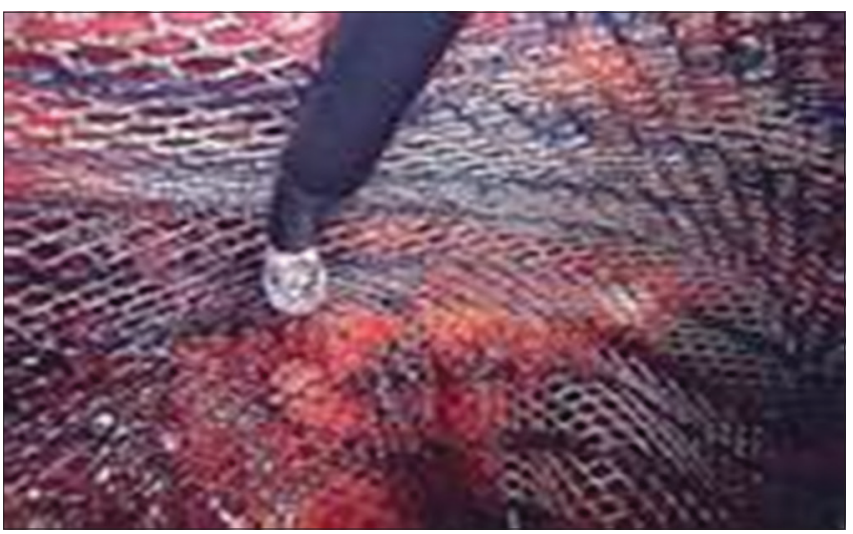

Figure 3: Mesh being tacked

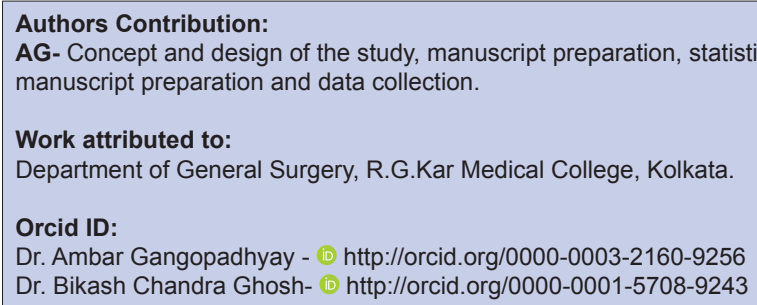
manuscript preparation and data collection.

Work attributed to:

Department of General Surgery, R.G.Kar Medical College, Kolkata.

Orcid ID:

Dr. Ambar Gangopadhyay - http://orcid.org/0000-0003-2160-9256

Dr. Bikash Chandra Ghosh- 1 http://orcid.org/0000-0001-5708-9243

AG- Concept and design of the study, manuscript preparation, statistical analysis, data interpretation, critical revision of manuscript; BCG- Review of literature,

Source of funding:: R.G.Kar Medical College, Kolkata.(All surgeries conducted in this Government Medical College).

Acknowledgement: Post-graduate Trainees and all staffs of Department of Surgery, R.G.Kar Medical College, Kolkata.

Conflict of Interest: None 